\title{
Ultrasonography and SARS-CoV 2 infection: a review of what we know and do not yet know
}

\author{
Sorin M. Dudea
}

Radiology Department, "Iuliu Hatieganu” University of Medicine and Pharmacy, Cluj-Napoca, Romania

The SARS-CoV 2 pandemic is testing the limits of the medical system, especially in areas with explosive disease development. Faced with the rapid evolution of the disease, the medical community has had to face multiple challenges: epidemiological triage, fast and early positive diagnosis, severity diagnosis, follow-up, early detection of complications, indicators of unfavorable evolution, multi-organ damage or signs of remission. On the other hand, in a combined effort of professional solidarity without borders, medical information has become very quickly accessible online (webinars, position letters, group discussions) without undergoing the much-needed test of publication validation.

Where does ultrasonography stand related to SARS CoV2 infection? This paper aims to present the current state of knowledge on how to apply the method for approaching patients in pandemic conditions.

Somewhat unexpectedly for a disease with predominantly lung damage, ultrasonography finds multiple applications, some incomplete and others not yet explored, in the management of the Covid19 patient.

The practice of the examination is hampered by the specific conditions of a highly contagious disease: the examiner requires special protective equipment, access to the patient is often made in improper conditions, in the emergency room or the intensive care unit, the machinery used is not always the best, examination time is limited, special precautions are required to decontaminate the equipment and especially the transducer, etc.

Received Accepted

Med Ultrason

2020, Vol. 22, No 2, 129-132

Corresponding author: Sorin M. Dudea

Radiology Department, "Iuliu Hatieganu"

University of Medicine and Pharmacy

3-5 Clinicilor street,

400006 Cluj-Napoca, Romania

Email: sdudea1@gmail.com
Benefiting from the fact that radiography is insensitive and non-specific and CT is more difficult to access for triage or for the examination of patients dependent on mechanical ventilation, the US finds its usefulness in evaluating SARS CoV2 patients especially due to its versatility.

Positive diagnosis. The US changes reported in SARS CoV2 lung infection are:

- B artifact - B line or lighthouse sign, often multiple, grouped, leading to the waterfall sign;

- loss of A line;

- pleural roughening / cobblestone;

- reduction of pleural sliding;

- discontinuous pleural line;

- subpleural consolidation - multifocal small nontranslobar or translobar, with or without mobile air bronchogram;

- posterior, basal and right-sided most frequent location;

- without pleural effusion or very rare incidence of it $[1,2]$.

It is noteworthy that these changes are not specific for COVID19 infection as they also occur in venous pulmonary hypertension / cardiogenic interstitial or alveolar edema or interstitial fibrosis. For COVID19, the heterogeneous mixture of $\mathrm{A}$ and $\mathrm{B}$ lines is characteristic and differs from pulmonary edema of cardiac origin, where a homogeneous increase of the B lines is observed [3]. As such, the appearance may be typical but not pathognomonic. US findings are interpreted in the clinical and epidemiological context of the patient.

A preliminary study demonstrated a good correspondence between B lines in US and ground-glass opacity (hyperdensity) foci at CT [4].

The sensitivity of US in the early diagnosis of SARS CoV2 infection is unknown [3]. The utility of bedside US in mild or moderate cases is reduced but the method 
is very useful in severe cases (5). Accessibility can be complemented by artificial intelligence (AI) protocols that highlight relevant changes for the ICU user who is not necessarily an expert in lung US [6].

Similar to the BIRADS classification, a COVID-CORADS classification for diagnostic purposes has recently been proposed, based on changes in chest CT [7]. The emergence of a US-COVID-CORADS classification is not unimaginable.

Triage. The US ability to highlight evocative changes for SARS CoV2 infection justifies the use of the method in the triage of symptomatic patients [8]. The method can benefit from miniaturized transducers (pocket or butterfly transducers) and can be useful in resource-limited settings.

For triage, a structured approach to the examination of the thorax in certain target areas may be useful [9], given the preferred location of the lesions posteriorly, basal and on the right side.

The transducers used are the usual, convextype for general abdominal use. The intercostal spaces are approached parallel to the ribs and the US beam is directed perpendicular to the pleura.

Starting with the US signs, institutional protocols are established in which the patient at risk and/or symptomatic and/or deficient in $\mathrm{O}_{2}$ saturation and who has positive US signs is considered COVID19 positive until proven otherwise and is tested and isolated until the RT PCR (reverse transcriptase polymerase chain reaction) result is obtained. In the absence of US signs, the likelihood that the patient's disease is not SARS CoV2 related increases. The described changes are valid only in the context of a massive SARS CoV2 epidemic, where, a priori, any patient with pulmonary symptoms is suspected of infection [3]. On the other hand, triage purpose at-home assessment of patients, with transducers / mobile devices, becomes possible.

Assessment of the severity of lung damage. For this purpose, combining the number of affected areas with the ultrasound appearance was proposed [10]. The ultrasound aspect defines lung damage as:

- Mild/moderate: B lines with increasing number and distribution; irregularity/interruption of the pleural line; heterogeneous mixture of $\mathrm{B}$ lines with A lines and normal pleural slip-spared areas; small subpleural consolidations $(<1 \mathrm{~cm})$;

- Severe: multiple B lines, also extending into the upper and anterior lung areas; confluent B lines; increase of the number and size of subpleural consolidations;

- Critical: coalescing B lines, extensive damage to the anterior and superior lung areas; multiple small consolidations in the anterior and superior areas; in the posterobasal areas, bilateral areas of alveolar filling syndrome - consolidation with or without aerial bronchogramappear; rare, small pleural collections, except for water overload of the patient.

Evolution. To express the severity of lung damage at a given time and to be able to assess the evolution of the disease, a severity score was imagined. A total of 12 lung areas are evaluated, each of which can receive a maximum of 3 points of severity so that the theoretical maximum score is 36 points [10]. Criteria for awarding points:

- A lines or insignificant $\mathrm{B}$ lines $=0$ points (normal);

- significant B lines (respectively more than 3 per intercostal space) $=1$ point;

- coalescent B lines with or without small subpleural consolidations $=2$ points;

- obvious, macroscopic, segmental or more extensive lung consolidation $=3$ points.

The proposed score has both the ability to express severity and to monitor the evolution and to guide the treatment with ventilation and posture [1]).

Alternatively, it was proposed to evaluate 14 lung areas, with the same 4 scores for each area, between $0=$ normal and $3=$ macroscopic lung consolidation [11].

The reappearance of A lines is found in convalescence [2].

Cardiac evaluation. Echocardiography allows the obtaining of valuable information on the volemic status. Five standard views are used for transthoracic echocardiography: apical 4 chambers, parasternal long axis, parasternal short axis, subcostal 4 chambers, subcostal through the inferior vena cava (longitudinal and transverse). In SARS-CoV2 infection, the heart changes consist of [12]:

- hyperdynamic cardiac function (increased cardiac output and LV ejection fraction);

- acute stress cardiomyopathy (Takotsubo) with segmental contraction abnormalities and apex ballooning;

- enlarged RV and acute pulmonary hypertension (increased RV postload and volume overload);

- late diffuse ischemic myocardial inhibition (insufficiency).

POCUS. In the ICU, the US ability to assess patients with severe lesions and to monitor their progress is maximized [13]. The chest examination in 6 areas is fast and acceptable. Examination in 12 areas is complete for assessing the condition of the lung [10].

US can evaluate the efficiency of bronchoscopy-type maneuvers, used to restore the bronchial patency [3]. 
US makes it possible to detect pneumothorax and pleural effusions. The association of pleural collection and SARS-CoV2 infection suggests heart failure.

It also becomes possible to evaluate the effectiveness of postural maneuvers to improve ventilation and guide pleural punctures [3].

Given the high incidence of thrombotic, arterial and/or especially venous complications in patients with COVID19 infection (14), it becomes reasonable to routinely use US to assess the venous circulation of the lower limbs in search of deep venous thrombosis.

Also, of interest is the potential for remote US operation performed on patients closed in isolated rooms (using an articulated, robotic arm) [3].

The disadvantages of US examination can be summarized as follows [3]:

- Prolonged exposure of the examiner to the risk of infection makes it necessary to use protective equipment that, in its turn, makes examination difficult;

- The US examination is performed in the COVID section (usually ICU), with on-site available devices and not in the ultrasound laboratory, with high-end machines;

- Impossibility of fluid disinfection of the ultrasound keyboard;

- Problematic disinfection of the transducers;

- The impossibility to keep the device uncontaminated traps the US machine inside the ICU / COVID ward;

- Interoperator reproducibility of the description of US changes is untested;

- There is still no standardization of how to report US changes.

There is a need to increase the professional competence of performing chest US of the personnel in the ICU wards, pulmonology departments and others [10] by performing a minimum of 25 supervised lung US examinations [15].

Progress. A preliminary report of 5 patients indicates the potential of CEUS in assessing the microcirculation of the abdominal viscera in severely impaired patients showing reduced arterial perfusion, uneven contrast distribution and areas with microinfarcts [16].

Unknown and work-in-progress:

- The role of Doppler or CEUS in the evaluation and prediction of peripheral circulatory status - why are diabetics more affected

- The role of Doppler or CEUS in the evaluation and prediction of renal impairment or deterioration of previously affected renal function (patients with nephropathy);
- The role of Doppler or CEUS in the evaluation and prediction of liver damage or deterioration of previously affected liver function (patients with liver disease);

- The role of Doppler and/or CEUS in the evaluation of cerebral microcirculation damage;

- Will sonoelastography ever play a role in this disease;

- Why in some patients the clinical onset of the disease is with acute abdominal symptoms and how abdominal ultrasound could help here;

- What would a US-COVID-CORADS look like for diagnosis or evolution monitoring?

The aspects observed with US are complex and supplemental to the clinical examination, indicating the usefulness of applying the method, especially in pandemic conditions [17].

The examination should be performed whenever necessary and for the benefit of the patient. If not necessary, the US exam should be postponed.

SARS CoV2 is here to stay for at least another year or so. Therefore, imaging diagnosis needs to be improved for the benefit of patient management. In pandemic conditions, it is expected for US to play a bigger role than RX or CT, not only in conditions of overload of the medical system but also in underdeveloped countries, as the only widely accessible imaging modality.

\section{References}

1. Xing C, Li Q, Du H, Kang W, Lian J, Yuan L. Lung ultrasound findings in patients with COVID-19 pneumonia. Critical Care 2020;24:174.

2. Peng QY, Wang XT, Zhang LN, Chinese Critical Care Ultrasound Study Group (CCUSG). Findings of lung ultrasonography of novel corona virus pneumonia during the 2019-2020 epidemic. Intensive Care Med 2020 Mar 12. doi:10.1007/s00134-020-05996-6.

3. Piscaglia F, Stefanini F, Cantisani V, et al. Benefits, Open questions and Challenges of the use of Ultrasound in the COVID-19 pandemic era. The views of a panel of worldwide international experts. Ultraschall Med 2020 Apr 15. doi:10.1055/a-1149-9872.

4. Poggiali E, Dacrema A, Bastoni D, et al. Can lung US help critical care clinicians in the early diagnosis of novel coronavirus (COVID-19) pneumonia? Radiology 2020 Mar 13. doi:10.1148/radiol.2020200847.

5. Lu W, Zhang S, Chen B, et al. A Clinical Study of Noninvasive Assessment of Lung Lesions in Patients with Coronavirus Disease-19 (COVID-19) by Bedside Ultrasound. Ultraschall Med 2020 Apr 15. doi:10.1055/a-1154-8795.

6. Noon C. A Ray Of Light: AI-Enhanced Ultrasound Is Helping Physicians On The Front Lines of COVID-19. GE Reports 2020 Mar 26. Available at:https://www.ge.com/ reports/the-good-fight-how-ai-enhanced-ultrasound-is- 
on-the-front-line-against-coronavirus/. Accessed May 02, 2020.

7. COVID working group of the Dutch Radiological Society. COVID-19 CORADS classification. Available at:https:// radiologyassistant.nl/chest/covid-19-corads-classification. Accessed May 02, 2020.

8. Fiala MJ. A Brief Review of Lung Ultrasonography in COVID-19: Is It Useful? - Ann Emerg Med 2020 Apr 8. doi:10.1016/j.annemergmed.2020.03.033.

9. Toma TP, Volpicelli G. Essential Image Acquisition Protocols for Thoracic Ultrasonography. Respiration 2020;99:231-238.

10. Smith MJ, Hayward SA, Innes SM, Miller ASC. Pointof-care lung ultrasound in patients with COVID-19 - a narrative review. Anaesthesia 2020 Apr 10. doi:10.1111/ anae. 15082 .

11. Soldati G, Smargiassi A, Inchingolo R, et al. Proposal for international standardization of the use of lung ultrasound for COVID-19 patients: a simple, quantitative, reproducible method. J Ultrasound Med 2020 Mar 30. doi:10.1002/ jum. 15285 .

12. Peng QY, Wang XT, Zhang LN; Chinese Critical Care Ultrasound Study Group (CCUSG). Using echocardiography to guide the treatment of novel coronavirus pneumonia. Crit Care 2020;24:143.

13. Lu W, Zhang S, Chen B, et al. A Clinical Study of Noninvasive Assessment of Lung Lesions in Patients with Coronavirus Disease-19 (COVID-19) by Bedside Ultrasound. Ultraschall Med 2020 Apr 15. doi:10.1055/a-11548795

14. Lodigiani C, Iapichino G, Carenzo L, et al. Venous and arterial thromboembolic complications in COVID-19 patients admitted to an academic hospital in Milan, Italy. Thromb Res 2020 Apr 23;191:9-14.

15. Arbelot C, Dexheimer Neto FL, Gao Y, et al. Lung Ultrasound in Emergency and Critically Ill Patients: Number of Supervised Exams to Reach Basic Competence. Anesthesiology 2020;132:899-907.

16. Jung EM, Stroszczynski C, Jung F. Contrast enhanced ultrasonography (CEUS) to detect abdominal microcirculatory disorders in severe cases of COVID-19 infection: First experience. Clin Hemorheol Microcirc 2020 Apr 23.doi:10.3233/CH-209003.

17. Buonsenso D, Para D, Chiaretti A. COVID-19 outbreak: less stethoscope, more ultrasound. Lancet Respir Med 2020 Mar 20. doi:10.1016/S2213-2600(20)30120-X. 